\title{
Hipertensión arterial: de la evidencia a las preferencias
}

\author{
Hypertension: from evidence to preferences
}

Juan Victor Ariel Franco ${ }^{a, b}$

Franco JVA. Hipertensión arterial: de la evidencia a las preferencias. Evid Actual Pract Ambul. 2019;22(4):e002039.

En este número de la revista se publican tres artículos relacionados a la hipertensión arterial (HTA): un resumen comentado de la nueva guía de HTA del Ministerio de Salud de la Nación Argentina ${ }^{1,2}$, un resumen comentado de una revisión sistemática Cochrane que analiza distintos objetivos de presión arterial ${ }^{3,4}$ y un resumen comentado de un ensayo clínico sobre la dosificación nocturna de antihipertensivos ${ }^{5,6}$.

La guía de HTA mencionada fue desarrollada luego de un arduo proceso metodológico que buscó integrar las pericias de los profesionales de la salud con la mejor evidencia científica, siguiendo los pasos de la medicina basada en la evidencia $(\mathrm{MBE})^{7}$. Esta guía propuso como metas terapéuticas una presión arterial sistólica (PAS) por debajo de $140 \mathrm{mmHg}$ y una presión arterial diastólica (PAD) por debajo de $90 \mathrm{mmHg}$ (recomendación fuerte), en concordancia con los hallazgos de la revisión sistemática comentada en este mismo número de Evidencia, que cuestiona los beneficios netos obtenibles con metas de presión arterial más bajas. Esta es una postura distinta de la American Heart Association, que disminuyó las metas a PAS/PAD $<130 / 80$ $\mathrm{mmHg}$, bajo la influencia del ensayo clínico SPRINT ${ }^{8}$. La guía argentina considera metas más estrictas según el riesgo cardiovascular y el riesgo de eventos adversos (recomendación condicional). Sin embargo, ninguna de las dos guías evaluó cuáles son los valores y preferencias de los pacientes a la hora de fijar metas terapéuticas más estrictas. Este sigue siendo el desafío de implementar integralmente el concepto de MBE: integrar la mejor evidencia disponible, las necesidades de información y pericia de los profesionales de la salud y los valores y preferencias de los pacientes. Algunas preguntas que pueden guiar este proceso podrían ser:

1) ¿Cuáles son las ventajas absolutas del descenso de presión arterial de 140/90 a 130/80? (e.g. número necesario a tratar, reducción de riesgo absoluto)

2) ¿Cuáles son los riesgos absolutos de potenciales eventos adversos asociados con metas más estrictas? (e.g. número necesario para dañar, incremento de riesgo absoluto)

3) ¿Cuál es el balance que los pacientes hacen de estos datos?

A modo de ejemplo, un estudio reciente en pacientes con multimorbilidad exploró el efecto de las preferencias de los pacientes adecuadamente informados sobre el balance neto en relación a las metas de presión arterial y encontró que: en el grupo etario de 50 a 64 años las preferencias de los pacientes sanos fue muy variable, algunos prefiriendo metas cercanas a $120 \mathrm{mmHg}$ y otros cercanas a $140 \mathrm{mmHg}$. En pacientes con antecedentes de accidente cerebrovascular, el balance se inclinó, paradójicamente, a metas más laxas. Sin embargo la distribución y variabilidad fue tan amplia que se sugirió evaluar la preferencia individual del paciente a la hora de definir las metas de presión arterial, usando el abordaje de toma de decisiones compartidas ${ }^{9}$.

Existen herramientas online que permiten indicar cuál podría ser la meta preferida basándonos en las preferencias individuales de los pacientes. ${ }^{10}$

Futuras ediciones de la guía nacional deberían incluir estas consideraciones en relación a la variabilidad de las preferencias en su recomendación condicional, idealmente incorporando estudios sobre las preferencias de pacientes locales de diversos estratos sociales y antecedentes clínicos.

\section{Referencias}

1. Salas J, Valente B, Valverdi R, Franco F. Nueva guía de hipertensión arterial en Argentina. Evid Actual Pract Ambul. 2019;22(04):2038-2038

2. Alcuaz MA, Rosende A. Guía de Práctica Clínica Nacional sobre Prevención, Diagnóstico y Tratamiento de la Hipertensión Arterial (HTA) 2019; 2019.

3. Saiz LC, Gorricho J, Garjón J, Celaya MC, Erviti J, Leache L. Blood pressure targets for the treatment of people with hypertension and cardiovascular disease. The Cochrane database of systematic reviews. 2018;7:010315. Available from: 10.1002/14651858.CD010315.pub3.

4. Sguiglia S. Objetivos de presión más bajos al estándar no reducirían la morbi-mortalidad en pacientes con hipertensión arterial y enfermedad cardiovas-cular. Evid Actual Pract Ambul. 2019;4:1101-1101.

5. Avendaño Y. El tratamiento de la hipertensión arterial a la hora de acostarse mejora la reducción del riesgo cardiovascular. Evid Actual Pract Ambul. 2020;22(04):2030-2030.

6. Hermida RC, Crespo JJ, Domínguez-Sardiña M, Otero A, Moyá A, Ríos MT, et al. Bedtime hypertension treatment improves cardiovascular risk reduction: the Hygia Chronotherapy Trial. European Heart Journal. 2019;Available from: 10.1093/eurheartj/ehz754.

7. Students4BestEvidence. What are the key steps in Evidence-Based Medicine?;

8. Whelton PK, Carey RM, Aronow WS, Casey DE, Collins KJ, Himmelfarb CD, et al.. 2017 ACC/AHA/AAPA/ABC/ACPM/AGS/APhA/ ASH/ASP $\mathrm{C} / \mathrm{NMA}$ /PCNA guideline for the prevention, detection, evaluation, and management of high blood pressure in adults a report of the American College of Cardiology/American Heart Association Task Force on Clinical pr. Lippincott Williams and Wilkins; 2018. Available from: 10.1161/HYP.0000000000000065.

9. Fletcher B, Hinton L, McManus R, Rivero-Arias O. Patient preferences for management of high blood pressure in the UK: A discrete choice experiment. British Journal of General Practice. 2019;69(686):629-637. Available from: 10.3399/bjgp19X705101.

10. Benefit harm balance of blood pressure targets; 2017. Available from: http://shiny.math.uzh.ch/user/wang/bha_htn [Last access: 2020/25/01].

\footnotetext{
a Departamento de Investigación, Instituto Universitario Hospital Italiano.

b Servicio de Medicina Familiar y Comunitaria, Hospital Italiano de Buenos Aires. juan.franco@hospitalitaliano.org.ar
} 